\title{
Inclusion of the $A S H 1$ gene that governs the neuroendocrine differentiation of lung epithelium as an additional prototypic 'lineage-survival oncogene'
}

\author{
Hirotaka Osada* and Takashi Takahashi \\ ${ }^{*}$ Division of Molecular Oncology, Aichi Cancer Center Research Insttute, 1-1 Kanokoden, Chikusa-ku, \\ Nagoya, 464-8681, Japan \\ Division of Molecular Carcinogenesis, Center for Neurological Diseases and Cancer, Nagoya University \\ Graduate School of Medicine, 65 Tsurumai-cho, Showa-ku, Nagoya 466-8550, Japan.
}

Corresponding to H.O. e-mail: hosada@aichi-cc.jp

We read the insightful review by Garraway and Sellers, which highlighted 'lineage dependency' as a new aspect of carcinogenic mechanism ${ }^{1}$, with great interest. The authors proposed the 'lineage-survival oncogene' concept and described the basic-helix-loop-helix (bHLH) transcription factor MITF as a prototype $^{2,3}$, and also speculated about other putative candidates. We would like to suggest the addition of another bHLH gene, achaete-scute homologue $1(\mathrm{ASH})$, as a prototypic lineage-survival oncogene on the basis of existing experimental evidence. Although pulmonary neuroendocrine cells (PNECs) that reside in the airway epithelium are suggested to have a role as a stem-cell niche for the lung $^{4}$, it has also been reported that gene-targeting of $A S H 1$ results in the loss of PNECs in the lung ${ }^{5}$, and the $A S H 1$ transgene promotes the development of lung tumours with a neuroendocrine feature in coordination with SV40 large-T antigen ${ }^{6}$. Further, suppression of $A S H 1$ expression by RNAi has been shown to induce cell-cycle arrest and apoptosis in vitro, as well as the inhibition of tumour growth in vivo in an ASH1-expression-dependent manner'. These findings support the 'lineage-survival oncogene' concept proposed by Garraway and Sellers, in that $A S H 1$ has a decisive role in the differentiation and survival of both normal and malignant cells of neuroendocrine lineage in the lung. Together, MITF and ASH1 seem to constitute what is at present a very limited group of prototypic lineage-survival oncogenes with sufficient supportive evidence. However, we anticipate that the list of lineage-survival oncogenes will expand considerably in the near future with solid evidence from experimental results.

1. Garraway, L. A. \& Sellers, W. R. Lineage dependency and lineage-survival oncogenes in human cancer. Nature Rev. Cancer 6, 593-602 (2006).

2. McGill, G. G. et al. Bcl2 regulation by the melanocyte master regulator Mitf modulates lineage survival and melanoma cell viability. Cell 109, 707-718 (2002).

3. Garraway, L. A. et al. Integrative genomic analyses identify MITF as a lineage survival oncogene amplified in malignant melanoma. Nature 436, 117-122 (2005).

4. Hong, K. U., Reynolds, S. D., Giangreco, A., Hurley, C. M. \& Stripp, B. R. Clara cell secretory protein-expressing cells of the airway neuroepithelial body microenvironment include a label-retaining subset and are criticalfor epithelial renewal after progenitor cell depletion Am. J. Respir. Cell Mol. Biol. 24, 671-681 (2001).

5. Borges, M. et al. An achaete-scute homologue essential for neuroendocrine differentiation in the lung. Nature 386, 852-855 (1997).

6. Linnoila, R. I. et al. Constitutive achaete-scute homologue-1 promotes airway dysplasia and lung neuroendocrine tumors in transgenic mice. Cancer Res. 60, 4005-4009 (2000).

7. Osada, H., Tatematsu, Y., Yatabe, Y., Horio, Y. \& Takahashi, T. ASH1 gene is a specific therapeutic target for lung cancers with neuroendocrine features. Cancer Res. 65, 10680-10685 (2005). 